\title{
Discrete particle simulation of shock wave propagation in a binary $\mathrm{Ni}+\mathrm{Al}$ powder mixture
}

\author{
D. Eakins and N. N. Thadhani ${ }^{\text {a) }}$ \\ School of Materials Science and Engineering, Georgia Institute of Technology, \\ Love Manufacturing Building, 771 Ferst Drive, Atlanta, Georgia 30332
}

(Received 4 October 2006; accepted 18 November 2006; published online 20 February 2007)

\begin{abstract}
Numerical simulations of shock wave propagation through discretely represented powder mixtures were performed to investigate the characteristics of deformation and mixing in the $\mathrm{Ni}+\mathrm{Al}$ system. The initial particle arrangements and morphologies were imported from experimentally obtained micrographs of powder mixtures pressed at densities in the range of $45 \%-80 \%$ of the theoretical maximum density (TMD). Simulations were performed using these imported micrographs for each density compact subjected to driver velocities $\left(U_{p}\right)$ of $0.5,0.75$, and $1 \mathrm{~km} / \mathrm{s}$, and the resulting shock velocity $\left(U_{s}\right)$ was used to construct the $U_{s}-U_{p}$ equation of state. The simulated equation of state for the $60 \%$ TMD mixture was validated by matching results obtained from previous gas-gun experiments. The details of shock wave propagation through the $\mathrm{Ni}+\mathrm{Al}$ powder mixtures were explored on several scales. It is shown that the shock compression of mixtures of powders of dissimilar densities and strength is associated with heterogeneous deformation processes leading to focused flow, particulation, and vortex formation, resulting in local fluctuations in pressure and temperature, which collectively are responsible for highly irregular "shock" fronts and unstable high-pressure states in granular media. (C) 2007 American Institute of Physics.
\end{abstract}

[DOI: $10.1063 / 1.2431682]$

\section{INTRODUCTION}

Granular compaction ${ }^{1-4}$ is an important processing technique used to produce a variety of materials, including pharmaceutical tablets, ${ }^{5}$ directionally aligned composite magnets, ${ }^{6}$ and nanostructured high-strength metal parts. ${ }^{7,8}$ Under dynamic shock-loading conditions, the process of granular compaction has been shown to initiate chemical reactions in reactive powder mixtures. ${ }^{9}$ Two classes of reactions have been proposed: ${ }^{10}$ shock-assisted, thermally induced reactions, which occur on the time scale of bulk temperature equilibration, and shock-induced reactions, where reaction is initiated within or shortly behind the shock front. While the mechanisms responsible for the thermally induced shock-assisted reactions are well established, those contributing to the ultrafast shock-induced reactions are not fully understood due to the inability to probe the processes leading to reaction initiation in real time (microsecond duration).

Shock wave compression of a granular media differs from that of homogeneous, full density solids. The process can result in extensive mechanical and chemical changes due to unique physical environments produced by void closure (e.g., particle comminution, heterogeneous deformation and flow, etc.) that are not observed in solids. It is challenging to probe these effects in real-time experiments, as the entire shock-compression event occurs over the span of several hundred nanoseconds, prohibiting the use of local imaging or spectroscopy. The true nature of the propagating shock wave is also difficult to characterize as the probes used (stress gauges, interferometry, etc.) provide response which is aver-

${ }^{a)}$ Electronic mail: naresh.thadhani@mse.gatech.edu aged over the area being resolved. Our understanding of the ultrafast physical, chemical, and mechanical processes that occur ahead and immediately behind the shock front can therefore only be progressed through modeling at the discrete particle level. Simulations of the compaction of powders and their mixtures can be used to develop and progress the understanding between experimentally observed macroscopic phenomena and the details of deformation at the particle level. This information is essential to probe the mechanochemical processes, long attributed to being responsible for the initiation of shock-induced reactions. ${ }^{9-11}$

Modeling of the shock-compression of granular systems has been performed by a number of investigators. ${ }^{12-22}$ However, still several questions remain unanswered. Some of these include the following.

(1) Does the shock wave propagate at a constant velocity over large distances? Is it appropriate to couple the experimentally measured shock velocity $U_{s}$ with the input stress $P$ to describe the shock-compression response of granular systems?

(2) How spatially and temporally heterogeneous is the shock front? What variations in rise time, pressure, and temperature can be expected within the front, and consequently can the propagating wave be truly classified as a "shock wave"?

(3) How can the type of mixing and mass flow within the shock front be best characterized? What mechanisms of mixing are activated for highly heterogeneous mixtures of constituents with large strength and density differences?

It is important to mention that while shock fronts are 
quite planar in homogeneous isotropic solids, the heterogeneous nature of granular systems and distribution of interparticle contacts give rise to a highly irregular and dispersed front. ${ }^{15,23}$ Stress can only be transmitted through interparticle contacts, ${ }^{23,24}$ which vary both spatially and temporally. Strictly speaking, such nonequilibrium behavior is not representative of a true shock, but a "catastrophic" shock, as suggested by Graham. ${ }^{25}$ The properties defined at the macroscale, however, are insensitive to these local inhomogeneities; there is little to distinguish between a shock wave and a nonequilibrium disturbance.

In the present investigation, discrete particle simulations of the shock compression of micron-scale $\mathrm{Ni}+\mathrm{Al}$ powder mixtures is performed using imported microstructures at starting densities of $80 \%, 60 \%, 52 \%$, and $45 \%$. Conventional terminology (shock and pressure) and symbolic representations $\left(P, U_{s}\right.$, and $\left.U_{p}\right)$ are used when describing features at the macroscale. Otherwise, alternative descriptors of inhomogeneous behavior are used to more appropriately detail the local character. The shock-compression response of the various $\mathrm{Ni}+\mathrm{Al}$ powder mixtures is characterized by applying single shocks at several velocities to determine the dependence of density on the shock and particle velocity $\left(U_{s}-U_{p}\right)$ relationship. The $U_{s}-U_{p}$ relationship calculated for the $60 \%$ TMD sample is validated against data obtained from gas-gun experiments performed previously under identical loading conditions. Furthermore, the shock front is tracked at multiple locations to investigate the modes of pressure transmission and ultimately characterize its heterogeneity.

In this paper, a brief review of the approaches used to model behavior at the mesoscopic level using simulated and real microstructures is presented first. The simulation procedure employed in the present work, including the process of importing experimentally obtained real microstructures and the setup of the model, is described next. Finally, the results of simulations and their validation along with observations of macroscopic and particle-level behavior are described and discussed.

\section{MODELING BACKGROUND}

Several studies of the effect of shock compression on local structure and thermally induced phase changes have been performed on microstructures generated using idealized geometries. The studies conducted by Williamson ${ }^{26}$ and $\mathrm{Ku}-$ mar et $a .^{27}$ have investigated shock energy generation/ dissipation during void closure in a regular close-packed array of spherical particles of uniform as well as varying size, and size distribution. While localized deformation and heat generation surrounding the voids have been demonstrated, the idealized geometries and distributions of particles and voids largely oversimplify the effects of compaction of a truly granular configuration.

More recently, real microstructures have been approximated through matching of microstructural statistics, such as size distributions, nearest neighbor distributions, and volume fractions. For example, in their work on the $\mathrm{Al}+\mathrm{Fe}_{2} \mathrm{O}_{3}$ + epoxy system, Austin et al. ${ }^{21}$ have demonstrated an annealing technique, which iteratively perturbs the locations of an initially random microstructure configuration until the desired nearest neighbor distribution converges upon statistics from experimental microstructures. Other methods have also been employed to approximate realistic conditions, such as the computationally efficient "pseudogravity" technique by Benson, ${ }^{15}$ which uses contact and sliding conditions to approximate the equilibrium position of particles as they are added sequentially to a system. Alternatively, Baer ${ }^{28}$ has generated randomly distributed, closely packed microstructures of cyclotetramethylene-tetranitramine (HMX-high melting explosive) crystals through a Monte Carlo/molecular dynamics algorithm, which increases the particle size of an equilibrated distribution of "nuclei" at a constant rate until the desired volume fraction or packing configuration is attained. While these approaches come closer to simulating the real microstructure, they still oversimplify the particle shape (which can be very irregular) and interparticle contacts. The greatest promise in capturing the highly varied features and configuration of heterogeneous systems, such as in powder mixture compacts, is in using real micrographs as the starting configuration for performing the simulations.

The use of imported information to solve microstructure level problems is not unique to the field of shock compression. Real micrographs have been imported for simulations to address a wide variety of problems including fluid flow, thermal conductivity, crack propagation, and mechanics of biological materials. For example, x-ray tomographs of asphalt mix have been used to simulate three-dimensional fluid flow and estimate permeability in pavements. ${ }^{29}$ The results indicated that the prior notions of a direct relationship between void volume fraction and permeability were incorrect. Microstructure-based simulations have also been used to determine the effect of processing on the thermal conductivity of thermal barrier coatings. ${ }^{30}$ Object oriented finite element meshes were constructed from two-dimensional (2D) micrographs of yttria-stabilized zirconia (YSZ) deposited by varying methods. Simulations utilizing the imported microstructures exhibited closer agreement with experimental results than a purely analytical approach. In the food industry, the linear elasticity and thermal conductivity of two-phase materials (polycrystalline ice, cream) have been simulated from X-ray computed tomography (CT) images. ${ }^{31}$ The dynamics of fracture and the influence of structure on the path of crack propagation have been investigated using imported microstructures of an $\mathrm{Al}+\mathrm{SiC}$ composite ${ }^{32}$ and $\mathrm{TiB}_{2}+\mathrm{Al}_{2} \mathrm{O}_{3}$ in the high strain-rate regime. ${ }^{33}$ Real microstructures are also being used to help solve problems in the area of human biology. Imported CT/magnetic resonance imaging (MRI) data have proven to be a very useful resource, from aiding in the simulation of brain tumor growth and its impact on surrounding tissues $^{34}$ to helping predict the stress distribution in feet during several stages of movement. ${ }^{35}$ In work more closely related to the subject matter of this paper, Benson has demonstrated the use of real microstructures to accurately reproduce the equation of state of copper through shock wave simulations. ${ }^{15}$ In short, imported microstructures allow more accurate predictions in systems that contain inherent inhomogeneities that can significantly influence the phenomena or processes being investigated. 
Granular mixtures consisting of mechanically diverse particles (metals versus ceramics versus polymers), as well as infinite variations in particle size distribution, morphology, and surface roughness, represent an ideal example of an inherently inhomogeneous system. Particle size ratio, morphology differences, volume fraction of constituents and voids, and nearest similar and dissimilar neighbor distributions are additional heterogeneous aspects that are introduced when considering multicomponent granular systems. Thus, the deformation and compaction of granular systems, which depend heavily on powder characteristics, can be generalized for idealized representations, but can only be truly captured using real, imported microstructures.

\section{SIMULATION PROCEDURE AND MODEL SETUP}

In the present work, discrete particle simulations of the shock compression of $\mathrm{Ni}+\mathrm{Al}$ powder mixtures were performed using imported microstructures obtained from scanning electron micrographs of compacts of varying initial density. The simulations were performed using the multimaterial Eulerian finite element code CTH (Ref. 36) developed by Sandia National Laboratories.

The compacts for obtaining imported microstructures were prepared by mixing nickel and aluminum powders of -325 mesh $(\sim 25 \mu \mathrm{m}$ average particle size $)$, in a 1:1 volumetric ratio, combined with Loctite Hysol epoxy, and pressing in a $\frac{1}{2}$ in. die to make $2 \mathrm{~mm}$ thick discs of $45 \%, 52 \%$, $60 \%$, and $80 \%$ density. The epoxy was added in amounts to fill only the void volume, so as not to affect the pressing. The cured discs were sectioned along their axis and the crosssectioned surface was imaged using scanning electron microscopy (SEM) to create montages spanning $1.62 \mathrm{~mm}$ in length and $261 \mu \mathrm{m}$ in width. Figure 1 shows the montages, aligned vertically in order of decreasing density. The brighter particles shown in the SEM montages are those of nickel, the grayish particles are aluminum, and the darker regions are the void space occupied by the epoxy filler. The halo effect noticed in the 45\% TMD sample around some of the particles is due to charging of the epoxy filler during SEM analysis. The montages of desired fields were acquired at sufficient resolution to capture the features of interest (in the present case, the irregular particle boundaries) and provide the source microstructures imported into the CTH code.

\section{A. Importing of real microstructures}

The procedure of importing the microstructure montage is summarized in the following steps.

(1) Manually threshold and separate each phase into distinct binary images.

(2) Construct vectorized images of each phase.

(3) Convert the resulting vectorized particle descriptions into the DIATOM compatible code.

The first step is most simply accomplished when the separate phases are easily identifiable. As shown in the SEM montages in Fig. 1, the large difference in atomic number of the aluminum and nickel phases gives rise to a high contrast in scattered electron energy. This difference can be exploited

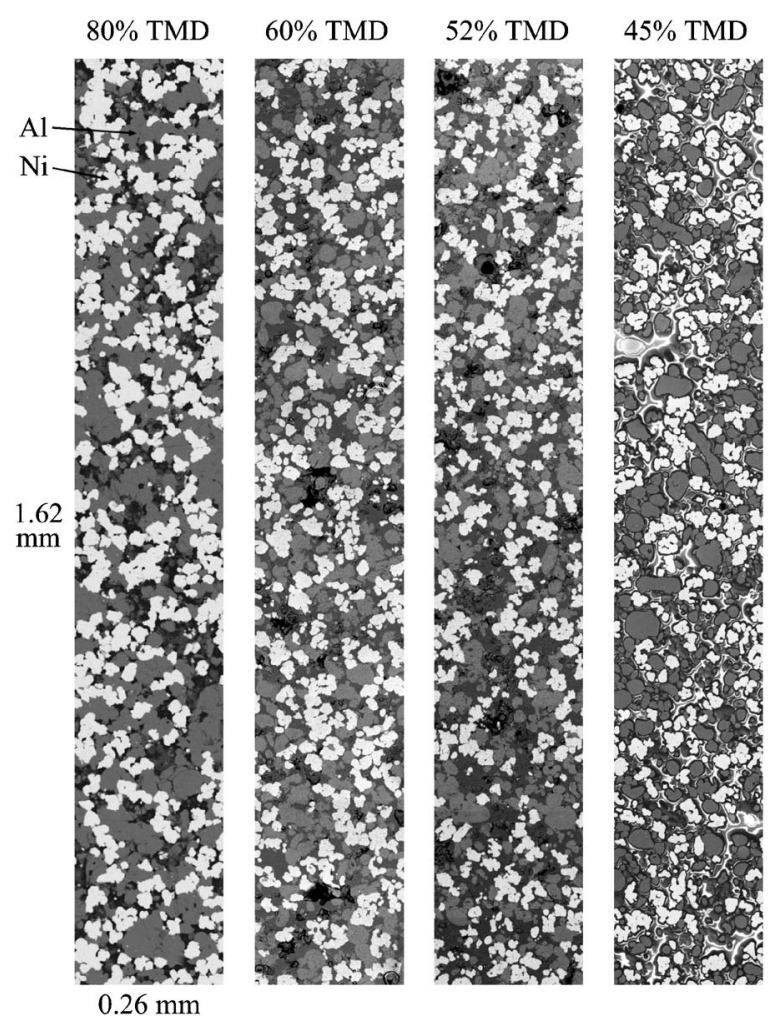

FIG. 1. Montages of $\mathrm{Ni}+\mathrm{Al}$ powder mixtures of varying density assembled from scanning electron micrographs of the sample cross section. The grayish particles are the aluminum, the brighter particles are the nickel, and the darker regions are the void space.

further using backscattered electron imaging. Simple thresholding algorithms can then be used to isolate each phase and construct binary images of high integrity. In order to preserve the discreteness of particles at the higher densities, it is sometimes necessary to manually "section" adjacent particles.

In the second step, the binary images of each phase are converted into vector graphics files (dxf format in the present case), which may be read by popular drafting packages or even graphics software such as ADOBE ILLUSTRATOR. Vectorization is the conversion of a raster image, in this case a rectangular array of binary values (0 or 255), into mathematical descriptions (points, lines, and polygons). In the case of particle vectorization, the particle boundaries are described by a collection of lines, arranged end to end. The resulting vector description will then be a sequence of points which, when joined, delineate the entire particle boundary. In the present work, vectorization was accomplished using Algolab's Raster to Vector conversion toolkit. ${ }^{37}$

The final step involves creating CTH compatible particle descriptions from the vector graphic information. For the particle descriptions considered in this paper, the vertices were transferred into DIATOM user defined shape (UDS) descriptions, as shown schematically in Fig. 2. The reformatting of the vertex data was automated, with the assignment of package tags and material identification handled through runtime inputs. This procedure can be similarly applied to construct part descriptions from dxf files for import into other codes [e.g., RAVEN (Ref. 38)]. 


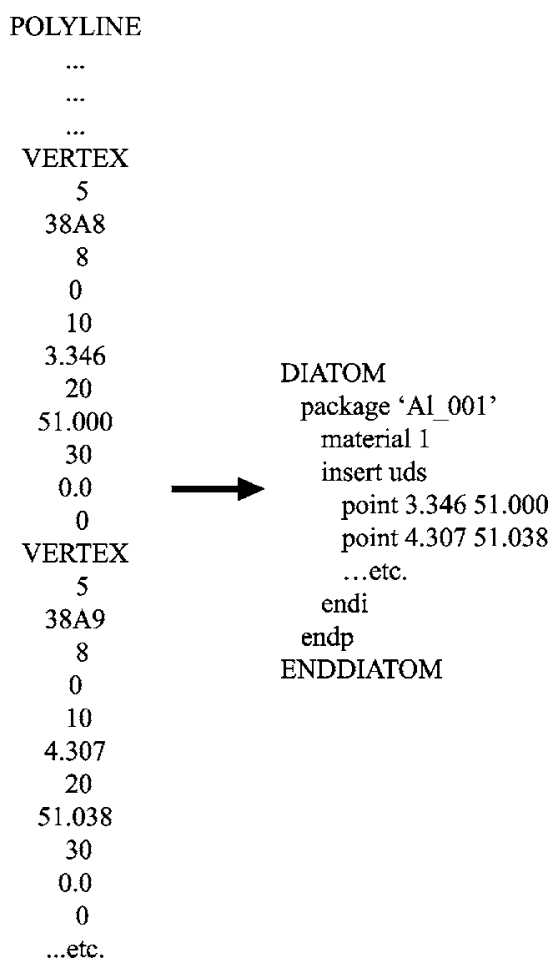

FIG. 2. Illustration of the conversion from a vectorized particle outline (left) to a CTH DIATOM description (right). The sequence of vertices in the vector graphic is easily identified and reformatted into a user defined shape (UDS) point list.

The starting configurations of the discrete particle models imported from microstructure fields at $80 \%, 60 \%, 52 \%$, and $45 \%$ TMD are shown in Fig. 3. In these configurations, the dark particles represent nickel and the gray contrast particles are aluminum. The white regions between the particles are unfilled and represent the void space.

\section{B. Model setup}

The simulations of shock wave propagation were performed on the imported microstructure fields shown in Fig. 3 . The shock was delivered by a copper piston moving upward from the bottom at initial particle velocities of 0.5 , 0.75 , and $1 \mathrm{~km} / \mathrm{s}$. This configuration was used to simulate the experimental setup which has been used in our prior work to measure the Hugoniot of $60 \%$ dense $\mathrm{Ni}+\mathrm{Al}$ powder mixtures. ${ }^{39}$ The lower and upper $y$ boundaries permit material to flow in/out, while periodic boundaries were prescribed on the left and right. While the edges of the microstructure fields could have been altered to yield true, seamless periodic boundaries, the placement and modification of overlapping particle extentions would be far too subjective and potentially disturbing to the true local particle arrangement at the edges. In order to use periodic boundaries, the calculation must use adaptive mesh refinement (AMR). When refining on boundaries, the overhead associated with AMR for a problem with many interfaces is very high and will run slower than a flat-mesh problem. Therefore, to keep memory requirements small yet still utilize periodic boundaries, the number of AMR blocks was kept at a minimum. The effec-

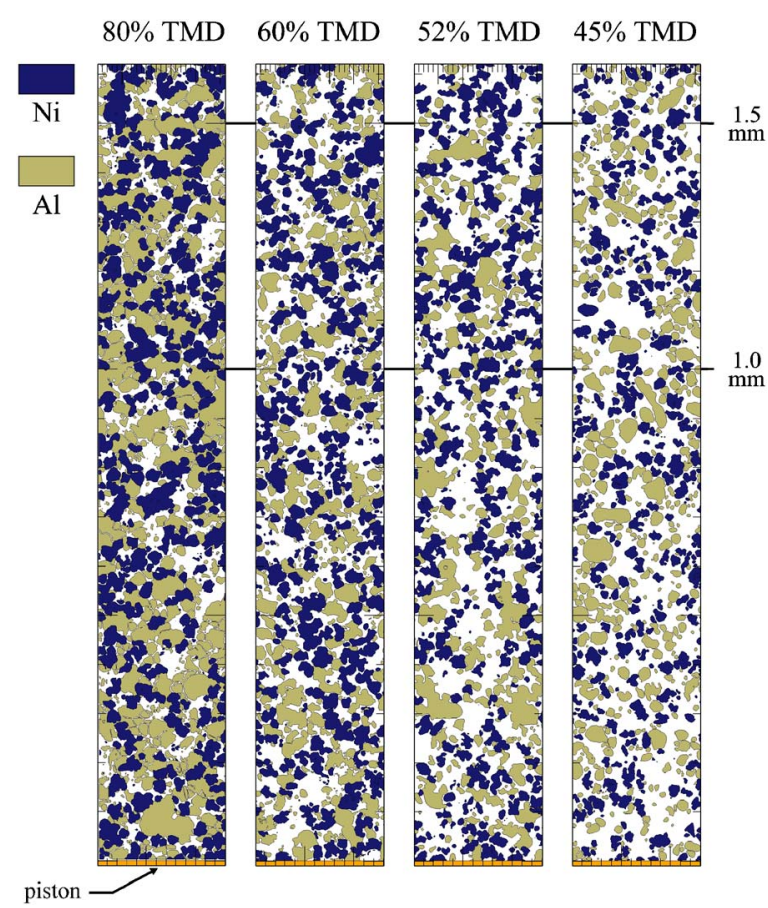

FIG. 3. (Color online) Discrete particles imported into CTH from the microstructure fields shown in Fig. 1. The contrast has been reversed to assist in visualizing particle deformation, the darker particles are now nickel, and the gray contrast particles are aluminum, with the white space illustrative of voids. A copper piston located at the bottom is used to deliver the assigned particle velocity and compresses the powders. The shock velocity is measured after propagation distances of 1.0 and $1.5 \mathrm{~mm}$, as indicated.

tive mesh size at the highest refinement level is $260 \times 1632$, which after considering the additional piston thickness yields an overall mesh resolution of $\sim 1 \mu \mathrm{m}^{2} /$ cell.

The shock properties for the nickel and aluminum particles and the copper piston were defined based on the MieGrüneisen equation of state. The constitutive behavior of nickel and aluminum was governed by the SteinbergCochran-Guinan model, ${ }^{40}$ while a Johnson-Cook viscoplastic model, ${ }^{41}$ modified to simulate a rigid material, was used for the copper piston. The strength of multimaterial cells was controlled by averaging the yield strengths by material volume fraction. Fracture of the constituents was not considered in the current models. In order to preserve the individual particle identities during deformation, several nickel and aluminum materials were defined and randomly assigned to their respective particles.

\section{Characterization of dynamic powder compaction}

The models described above are ideal for probing the phenomenon of shock compression of powder mixtures at multiple length scales. At the macroscale, the large model domain permits estimations of bulk properties (significant and applicable to experimental results). Furthermore, the models possess the resolution (discrete representation of particles) to explore the micromechanical effects at the particle level.

In the present work, the shock velocity was determined based on the arrival of the shock wave (profile monitored through entire sample width) at two spatial locations, corre- 
sponding to travel through distances of 1.0 and $1.5 \mathrm{~mm}$ (Fig. 3 ). The time at which a region of the profile reached the peak high-pressure state at these locations was taken as the arrival time. The two measures were used to determine the constancy of the shock velocity over the distances probed.

An in-depth particle-level characterization of the inhomogeneous nature of shock compression in granular systems was also accomplished. Measures of the spatial and temporal dispersion of the advancing peak stress front across the sample width were obtained by monitoring its irregularity. The heterogeneity of pressure and temperature following void collapse was also noted. Finally, the complex mechanisms of deformation and their evolutions, specific to each component, were characterized from sequences of images captured at different times.

\section{RESULTS OF MODEL SIMULATION}

Since shock compression of granular media is a multilevel phenomenon, the findings of this paper are presented in the order of diminishing spatial scale. First, macroscopic observations, analysis, and validation of bulk shock properties are presented. Next, the shock front behavior and its heterogeneous character are discussed. Finally, elements of local mesoscopic particle deformation and mixing between dissimilar constituents are characterized.

\section{A. Analysis of bulk properties and validation with experiments}

Using the method described earlier, the velocity of the shock wave propagating through $\mathrm{Ni}+\mathrm{Al}$ mixtures of varying initial density (80\%-45\% TMD) was determined for each of the three corresponding particle velocities $(0.5,0.75$, and $1.0 \mathrm{~km} / \mathrm{s})$. The shock velocity $\left(U_{s}\right)$ for each density mixture plotted against particle velocity $\left(U_{p}\right)$ is shown in Fig. 4. The intercept and slope of the linear $U_{s}-U_{p}$ plot yield, respectively, the equation-of-state parameters $C_{0}$ (sound speed) and $S$ over the region investigated. These parameters are listed in Table I as a function of density. As noted by Benson et al. ${ }^{38}$ and Borg et al., ${ }^{42}$ the powder compact initial density has the greatest influence on sound speed. However, over the range of densities investigated in our work, the change in slope $S$ is also seen, although it is less pronounced. The dependence of sound speed $C_{0}$ and $S$ on density can be expressed as

$$
\begin{aligned}
& C_{0}\left(\rho_{00}\right)=0.0002 \rho_{00}^{2}-0.0078 \rho_{00}+0.0579, \\
& S\left(\rho_{00}\right)=0.0178 \rho_{00}+0.7724 .
\end{aligned}
$$

The validity of the simulated equation-of-state parameters listed in Table I has been evaluated through comparison to experimental data collected previously by the authors. ${ }^{39,43}$ Impact experiments were performed on equivalent $\mathrm{Ni}+\mathrm{Al}$ powder mixtures at an initial density of $60 \%$ TMD. Piezoelectric polyvinylidene fluoride (PVDF) stress gauges, with an approximate active area of $9 \mathrm{~mm}^{2}$, were used to measure stress profiles entering (input) and exiting (propagated) the powder sample. Figure 5 shows an example of such inputand propagated stress profiles corresponding to a particle velocity of $0.79 \mathrm{~km} / \mathrm{s}$. The shock velocity $U_{s}$ was determined

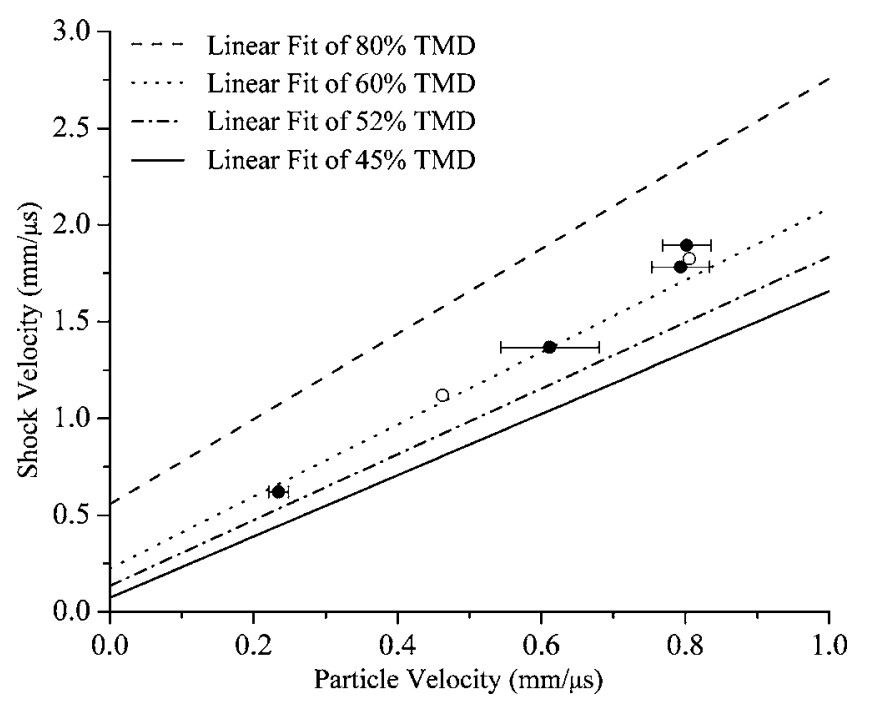

FIG. 4. Shock velocity-particle velocity $\left(U_{s}-U_{p}\right)$ relationship predicted from simulations as a function of density (lines). The initial density has a large influence on the sound speed (intercept), though only modest changes in the slope. Also shown are experimental data obtained from a $60 \%$ TMD Ni $+\mathrm{Al}$ powder mixture. Open circles are data for which only the shock velocity was obtained and the input pressure calculated. The error in particle velocity shown is determined from sample thickness, shock arrival time, and pressure uncertainties. Maximum error in shock velocity of $\pm 0.02 \mathrm{~mm} / \mu \mathrm{s}$ is omitted for readability.

from the shock arrival time in each gauge record corresponding to the travel time through the sample thickness (nominally $3.85 \mathrm{~mm}$ ) and coupled with the measured input stress to establish the high-pressure state of the $60 \%$ dense $\mathrm{Ni}$ + Al mixture. Use of the jump conditions permitted the conversion of the measured stress versus shock velocity states to $U_{s}-U_{p}$ states. The experimentally measured $U_{s}-U_{p}$ data for the $60 \%$ dense $\mathrm{Ni}+\mathrm{Al}$ mixture are also shown along with the simulated $U_{s}-U_{p}$ states in Fig. 4 to provide validation of the model simulation.

\section{B. Constancy of shock velocity}

The experimental determination of the high-pressure shocked state relies on coupling of the measured input stress and apparent shock velocity through the given sample thickness. The validity of this coupling relies on the assumption that the shock velocity is steady (unchanging), which is considered to be true for solids and/or overdriven shocks. The same assumption may not be appropriate for highly porous solids (powders), where energy dissipation in closure of voids and surface interactions are expected to cause significant dispersion of the shock front. To investigate this, the shock velocity was determined at two locations correspond-

TABLE I. Equation-of-state parameters as a function of density.

\begin{tabular}{ccc}
\hline \hline $\begin{array}{c}\text { Initial density } \\
(\% \mathrm{TMD})\end{array}$ & $\begin{array}{c}C \\
(\mathrm{~km} / \mathrm{s})\end{array}$ & $S$ \\
\hline 80 & 0.5667 & 2.1853 \\
60 & 0.2242 & 1.8630 \\
52 & 0.1363 & 1.6978 \\
47 & 0.0796 & 1.5929 \\
\hline
\end{tabular}



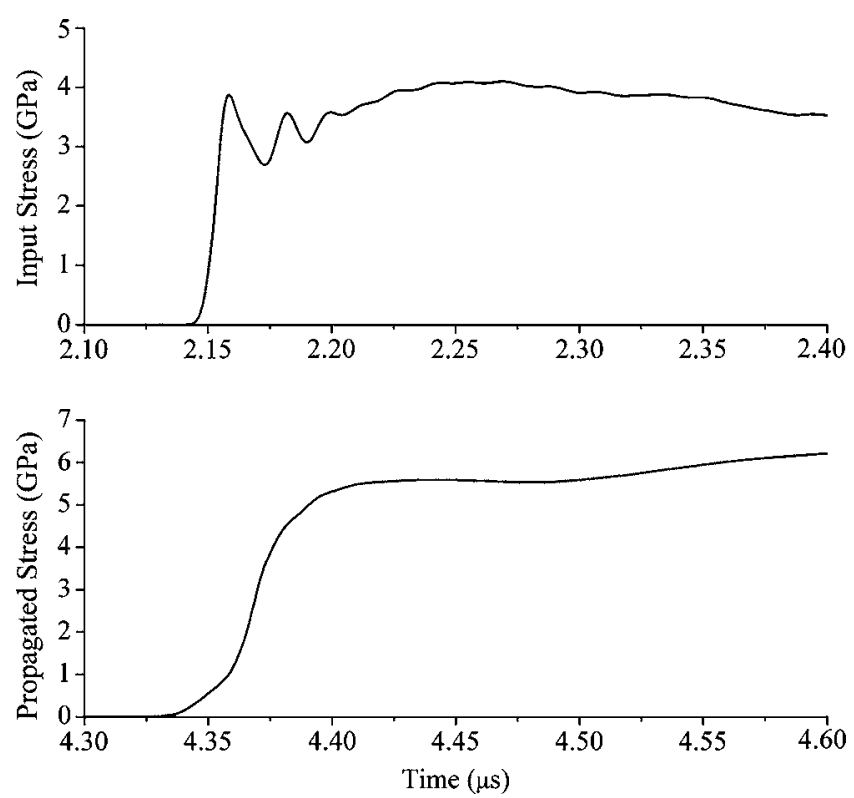

FIG. 5. Example of input and propagated stress traces obtained from a parallel-plate impact experiment performed on a $60 \% \mathrm{TMD} \mathrm{Ni}+\mathrm{Al}$ powder mixture impacted at $0.92 \mathrm{~km} / \mathrm{s}$. The reverberations in the input trace are due to multiple reflections through the low impedance gauge package. Note the difference in rise times between the input $(\sim 10 \mathrm{~ns})$ and propagated ( $\sim 40 \mathrm{~ns}$ ) traces.

ing to propagation distances of 1.0 and $1.5 \mathrm{~mm}$ for the $80 \%$, $60 \%$, and $45 \%$ TMD models. The results yielded nearly similar measures of $U_{s}$, for each case, with discrepancies within the error associated with locating the position of a nonplanar front.

\section{Shock front character}

While necessary for validation, the simulated $U_{s}-U_{p}$ relationships for the powder compacts investigated only describe a bulk response and give no indication of the complex structure of the shock front in granular media. A deeper understanding of the mechanisms governing shock wave propagation can be gained through examination of pressure surfaces constructed in the spatial domain. Snapshots of the shock front propagating at $U_{p}=1 \mathrm{~km} / \mathrm{s}$, through the entire $\sim 250 \mu \mathrm{m}$ sample width, are shown in Figs. 6(a)-6(c) for arbitrary locations within the $80 \%, 60 \%$, and $45 \%$ TMD mixtures. In the $80 \%$ and $60 \%$ TMD mixtures, the highpressure state is preceded by a broad low-amplitude stress network. This so-called "network" comprises the effects of an irregular array of elastically loaded particles and stressfree voids, the extent of which is as much as $300 \mu \mathrm{m}$ ahead of the peak-pressure front in the $80 \%$ TMD mixture [Fig. $6(\mathrm{a})]$. In the $60 \%$ TMD mixture, this network only spans about $100 \mu \mathrm{m}$ [Fig. 6(b)] and is entirely absent in the 45\% TMD mixture [Fig. 6(c)]. If these features are considered analogous to "elastic precursor" and "plastic" waves in solid materials, then these observations suggest that the disturbances shown are similar to the structure of "underdriven" shocks. When viewed at several locations, it becomes clear that the separation distance between the elastic network and peak-pressure front is constant. This suggests that a balance is obtained between elastic loading from the peak-pressure

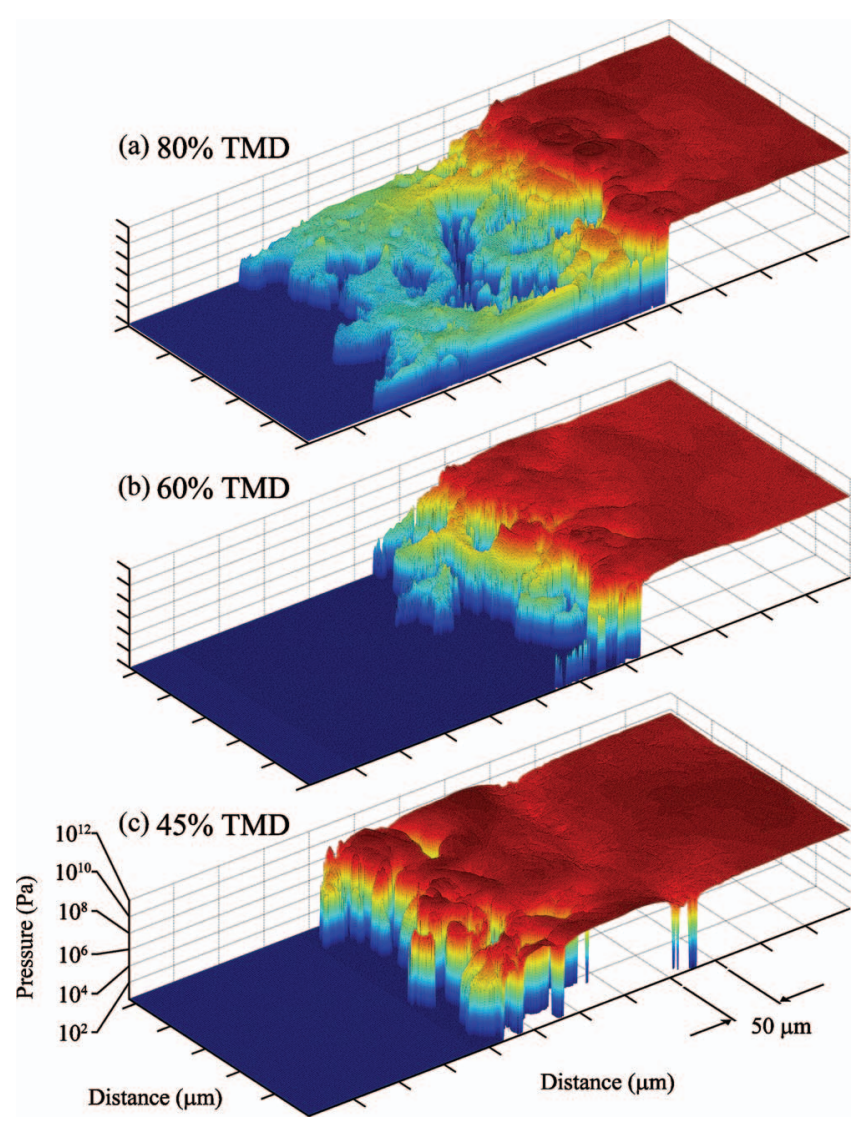

FIG. 6. (Color) Pressure surfaces in the spatial domain constructed for the (a) $80 \%$, (b) $60 \%$, and (c) $45 \%$ TMD simulations. Scaling is equivalent for all plots. The logarithmic pressure scale brings out the inhomogeneity of the elastic front network and shock front. Note the suppression of an elastic front network and the increased presence of release pockets (holes in the pressure surface) in the low (45\% TMD) initial density compact.

front and unloading from the void regions that is proportional to the apparent shock velocity (which in turn is governed by the void content).

The influence of porosity does more than simply diminish the low-amplitude stress network. As the initial density decreases (or porosity increases), a significant change in the stability of the high-pressure state is observed. At 45\% TMD, the presence of large void regions has the effect of introducing pronounced instabilities in pressure. This is evidenced by the many release pockets within the peak-pressure front, as shown in Fig. 6(c). The effect of these pockets is most clearly evident in the pressure profiles shown in Figs. 7(a)-7(c). The profiles are the pressure-time history averaged over the model sample width, obtained at a fixed location. Linear scaling of the pressure axis deemphasizes the elastic stress network (seen in Fig. 6), focusing instead on the highpressure zone. Perhaps the most striking feature of the pressure-time histories is the variability in pressure beyond the high-amplitude front. Clearly, a steady-state wave is not achieved at the particle level. Estimates of the time needed to attain the peak-pressure state (rise time) are also shown. As expected, lower density powder compacts exhibit longer rise times, apparently due to release events occurring within the high-amplitude front.

It stands to reason that the instabilities being observed are the micromechanical fluctuations responsible for the bulk 

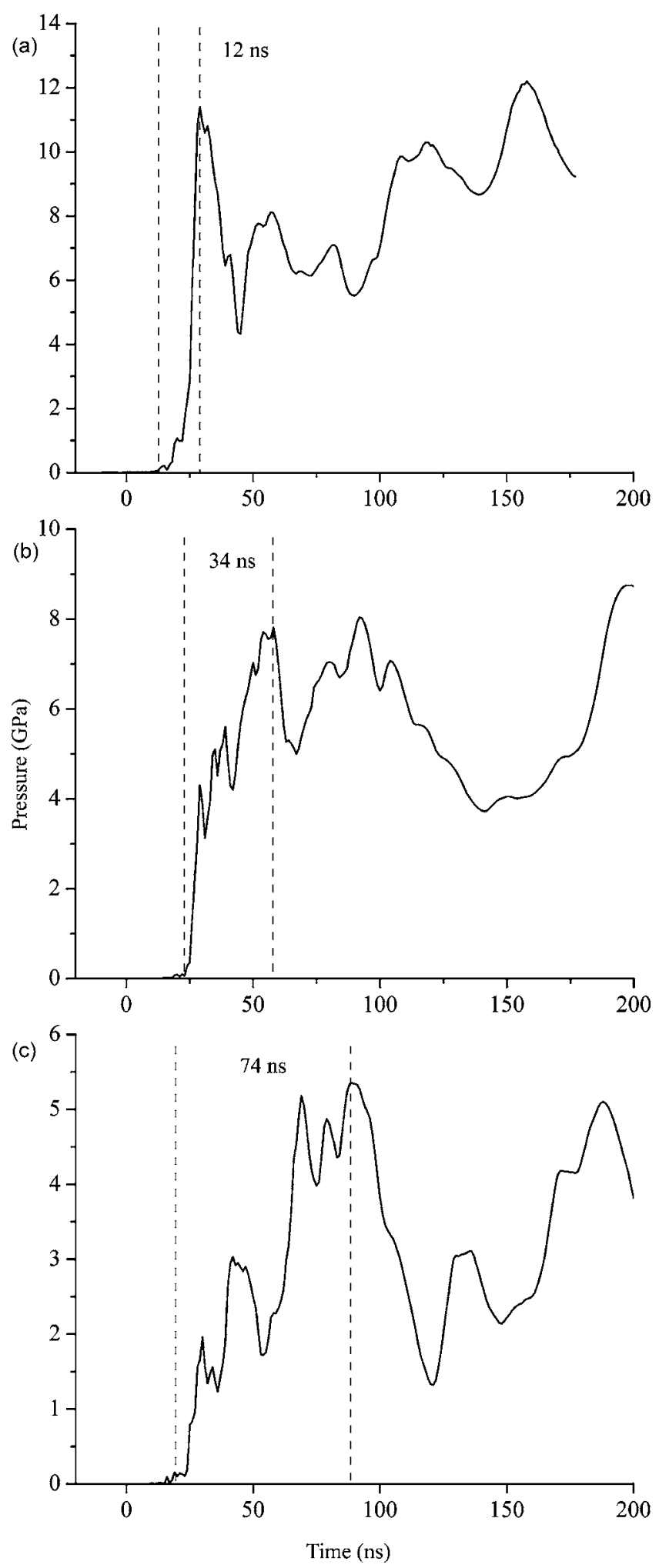

FIG. 7. Pressure-time histories for (a) $80 \%$, (b) $60 \%$, and (c) $45 \%$ TMD mixtures averaged over model sample width obtained at a fixed location showing the effects of release pockets on the variability in pressure beyond the high-amplitude front. Estimates of the interval between the arrival of the front and the first peak pressure indicate longer "rise times" with increased porosity.

effect known as "shock wave front dispersion." This effect, characterized by the temporal widening of the shock front, is observed regularly in porous materials ${ }^{44,45}$ and is most clearly seen in stress profiles captured following shock propagation through powders, as illustrated in the propagated gauge record shown in Fig. 5. The propagated stress trace climbs smoothly to the peak, over the span of $90 \mathrm{~ns}$. Whereas line plots averaged over a $261 \mu \mathrm{m}$ width were used to construct the pressure histories shown in Fig. 7, PVDF stress gauges sample a much larger region of material (9 $\mathrm{mm}^{2}$ area). Thus, the smooth ramp to peak pressure measured by the gauges (shown in the profiles in Fig. 5) is the result of averaging the response of many hundreds of particles, which dampens the local fluctuations caused by release pockets. The equilibration times listed above are characteristic of the more familiar notion of rise times of shock profiles measured in highly porous solids.

The elastic stress network structure and separation distance between the low- and high-amplitude fronts are due to processes occurring before significant densification. Once the voids have been closed, i.e., the material is well within the high-amplitude stress regime, the local pressures and temperatures attained can also be shown to depend highly on the piston (particle) velocity and initial density. The highpressure portion of the shock front observed at an arbitrary position in each mixture of different initial densities is shown in Fig. 8, as a function of particle velocity. The spatial irregularity of the front appears to be independent of impact velocity and spans approximately the diameter of a particle $(\sim 25 \mu \mathrm{m})$. It is worthwhile to note that while irregularities on the order of a particle diameter can be observed at any given time, these fluctuations are not sustained. Rather, the regularity in inhomogeneity ensures that particle configurations conducive to front advancement or delay are distributed evenly. The cell pressures predicted for the different mixtures also depend heavily on the starting density and particle velocity, with greater ranges in local pressure observed at the higher velocities and initial densities.

Cell temperatures calculated for the same fields are presented in Fig. 9. Again, the effect of particle velocity is apparent, with the highest local temperatures produced at $1 \mathrm{~km} / \mathrm{s}$ in all cases. While high local pressures appear to be concentrated at impact sites between adjacent confined particles, local temperature spikes are observed in the regions of high shear and mass flow. Not surprisingly, the lowest density microstructure field, which possesses the largest void volume available for material deformation and flow, exhibits the highest local temperatures for any given particle velocity.

\section{Deformation and mixing mechanisms}

One particular advantage of using experimentally acquired images to define the initial mixture configuration is the potential of simulating the actual, complex, particle-scale deformation modes activated during shock compression which are otherwise difficult to observe from experiments even with available time-resolved diagnostic techniques. In contrast, the states computed using idealized (simulated) microstructures/geometries lack the heterogeneities necessary to reproduce the details of localized deformation and mass flow. These omitted details become increasingly significant as the disparity between simulated and real powder mixtures grows (e.g., spherical versus highly asymmetrical 

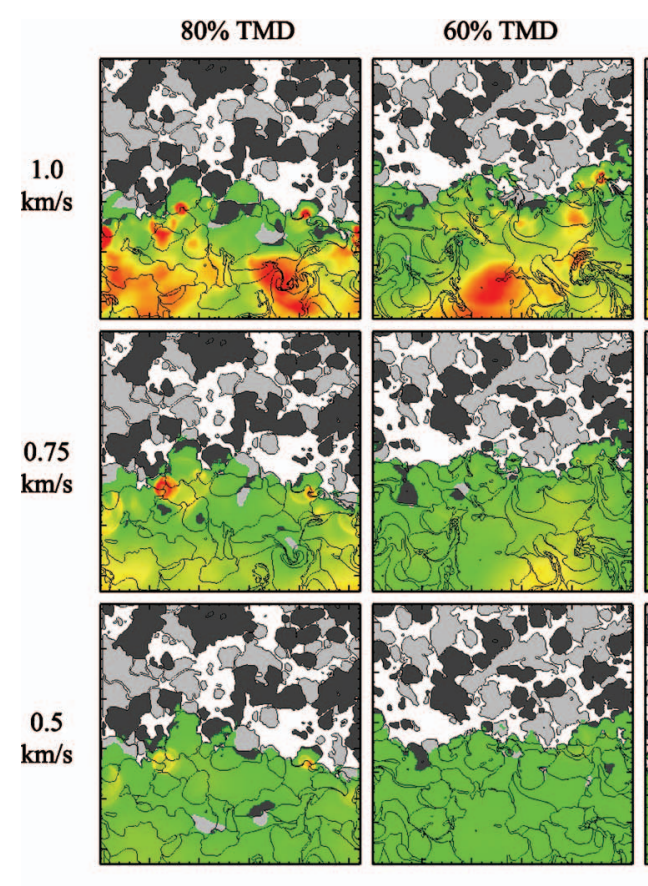

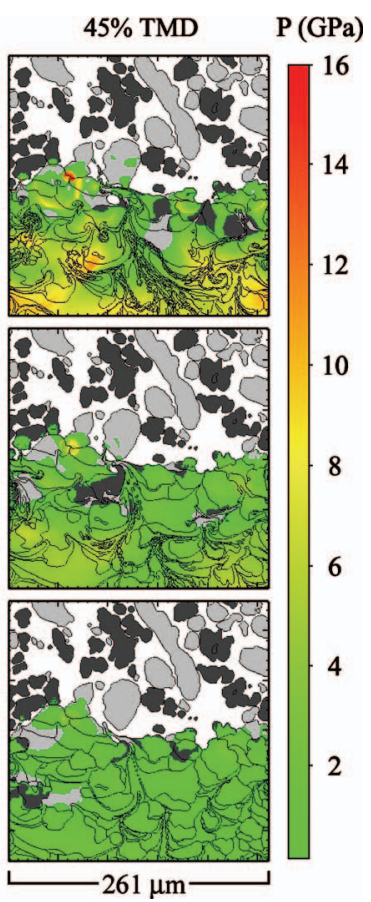

FIG. 8. (Color) Images showing cell pressure as a function of particle velocity and starting density at a fixed location. With increasing density, the particles become confined, leading to the development of high local pressures. particles, ordered versus random packing, etc.). This section will first address the observed mesoscale features of powder compaction, followed by an analysis of the deformation mechanisms dominating this process.

A comparison between the micrographs of the end states of the $60 \%$ TMD simulation $\left(U_{p}=0.5 \mathrm{~km} / \mathrm{s}\right)$ and a recovered specimen shock compressed at nearly the same conditions $\left(U_{p}=0.55 \mathrm{~km} / \mathrm{s}\right)$ is presented in Figs. 10(a) and 10(b). One can qualitatively conclude that the microstuctures appear quite similar, showing the preferential deformation of the dark contrast aluminum particles, the grouping of white contrast nickel particles into comparatively sized clusters, and the global bowing of particles in the direction of shock propagation. It can also be seen that the degree of aluminum deformation is slightly exaggerated in the simulation [Fig. 10(a)], most likely due to the absence of out-of-plane flow restrictions associated with two-dimensional models.

The three dominant mechanical phenomena observed in the simulations that contribute to local plastic deformation, energy dissipation, and overall propagation of the compressed zone are focused flow, particulation, and vortex formation. Focused flow (often interpreted as "jetting") describes the local deformation of material at enhanced velocities through a restricted passage. Examples of singular and cooperative focused flows are shown in snapshots at different times, as illustrated in Figs. 11(a) and 11(b). A singular focused flow is observed [Fig. 11(a)] when a compressed aluminum particle (marked $\mathrm{A}$ ) is forced through an opening

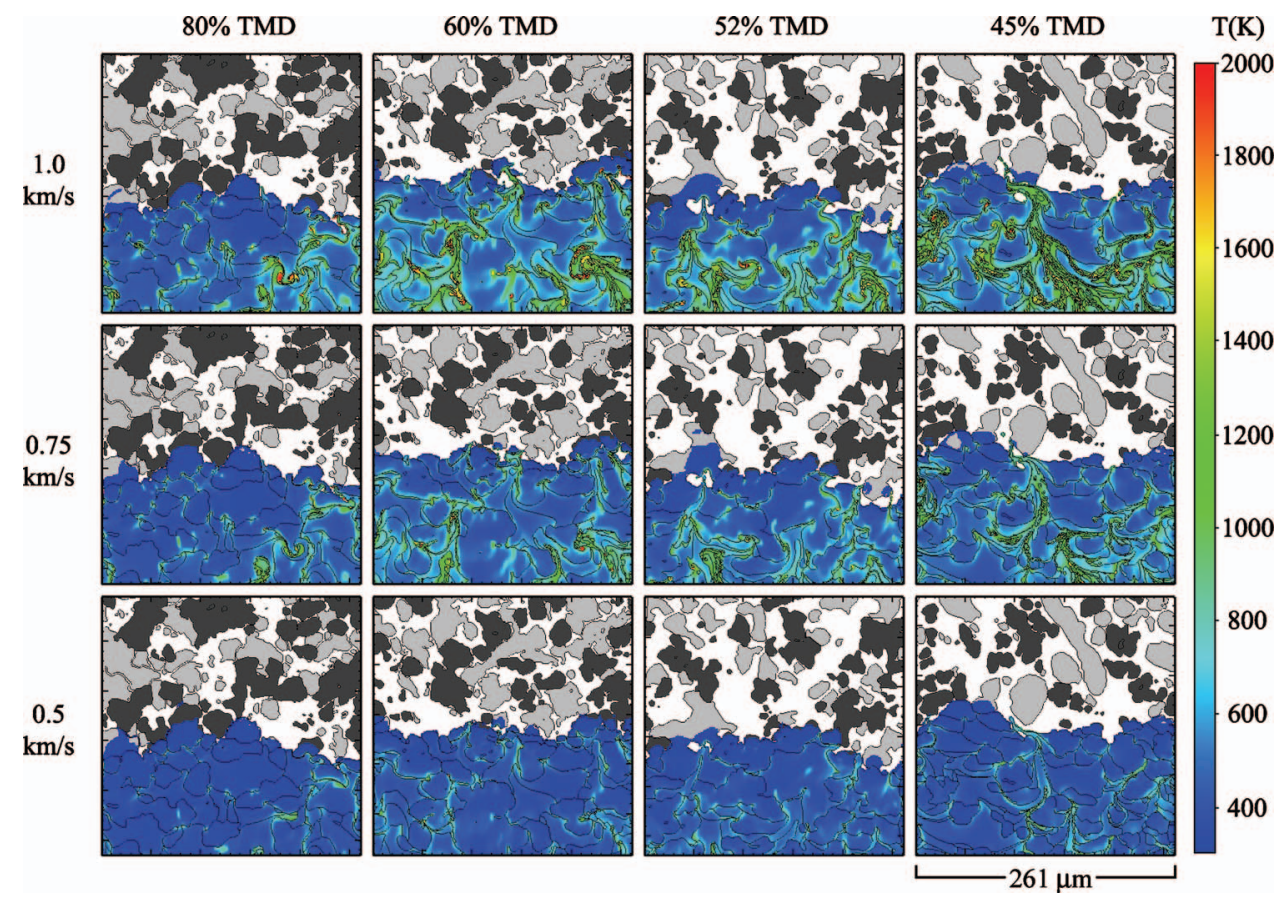

FIG. 9. (Color) Images showing cell temperature as a function of particle velocity and starting density from the same location as in Fig. 7. In contrast to local pressures, the highest local temperatures are developed in the regions of high mass flow, which are more predominant in the $45 \% \mathrm{TMD}$ model. 

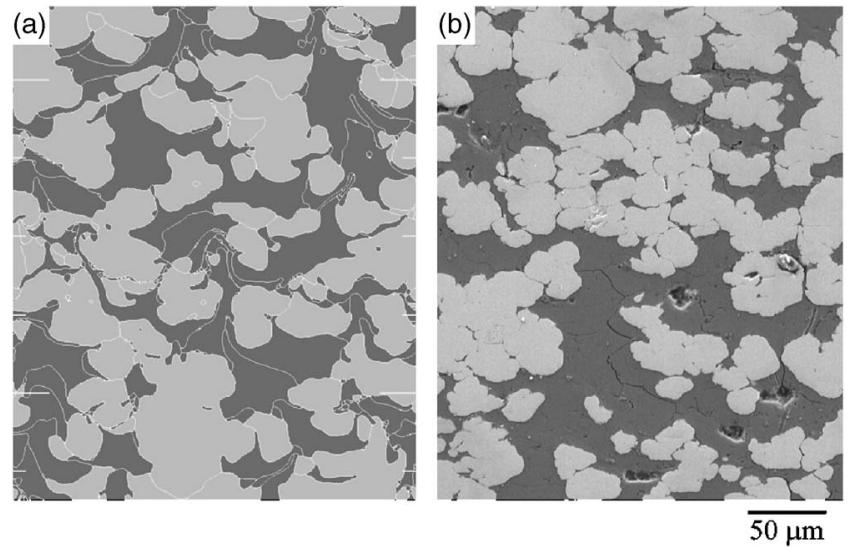

FIG. 10. Final, end-state micrographs of a $60 \%$ TMD Ni+Al mixture obtained (a) from simulation at $U_{p}=0.5 \mathrm{~km} / \mathrm{s}$ and (b) experimentally with $U_{p}=0.55 \mathrm{~km} / \mathrm{s}$. Qualitative comparison illustrates that the simulation predicts a dynamically deformed state structure quite similar to that obtained from experiments.

between adjacent dark contrast nickel particles. The resulting flow is the source of increased velocity $(>3 \mathrm{~km} / \mathrm{s})$, and high localized temperature and pressure. Cooperative focused flow [Fig. 11(b)] occurs when multiple aluminum particles (marked $\mathrm{P}, \mathrm{Q}$, and $\mathrm{R}$ ) are simultaneously forced through the same nickel barrier. The temperatures produced in regions of cooperative focused flow are often greater than those in regions of singular focused flow. Furthermore, due to their size, cooperative flow commonly dictates the extent of deformation in adjacent material.

Both forms of focused flow are responsible for the crescent-shaped profiles of the nickel particles observed in Figs. 10(a) and 10(b). Severe focused flow of Al through $\mathrm{Ni}$ particles can also lead to particulation. Shown in the series of snapshots in Figs. 12(a)-12(f) are the events leading to particulation of Ni particles (labeled 1 and 2) from the cooperative focused flow of Al. As mentioned earlier, fracture/ fragmentation modes of failure were not incorporated in the constitutive models used in this work, and hence are not responsible for the particulation of nickel. The model as-

(a)

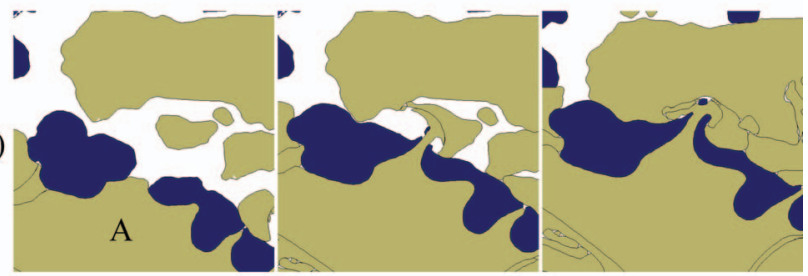

(b)

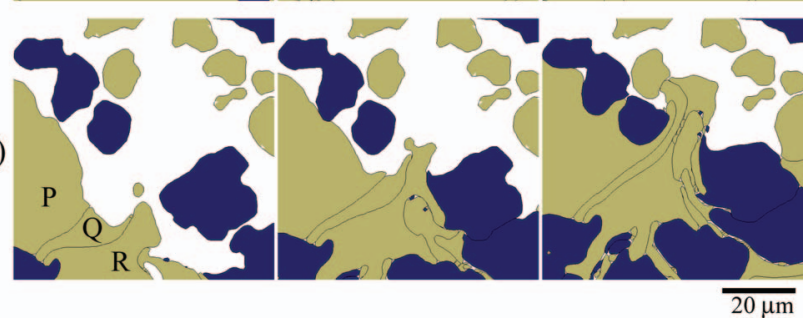

FIG. 11. (Color) Examples of (a) singular and (b) cooperative focused flows of aluminum through nickel barriers which can be a source of increased velocity and high localized temperature and pressure. Time between frames is $10 \mathrm{~ns}$

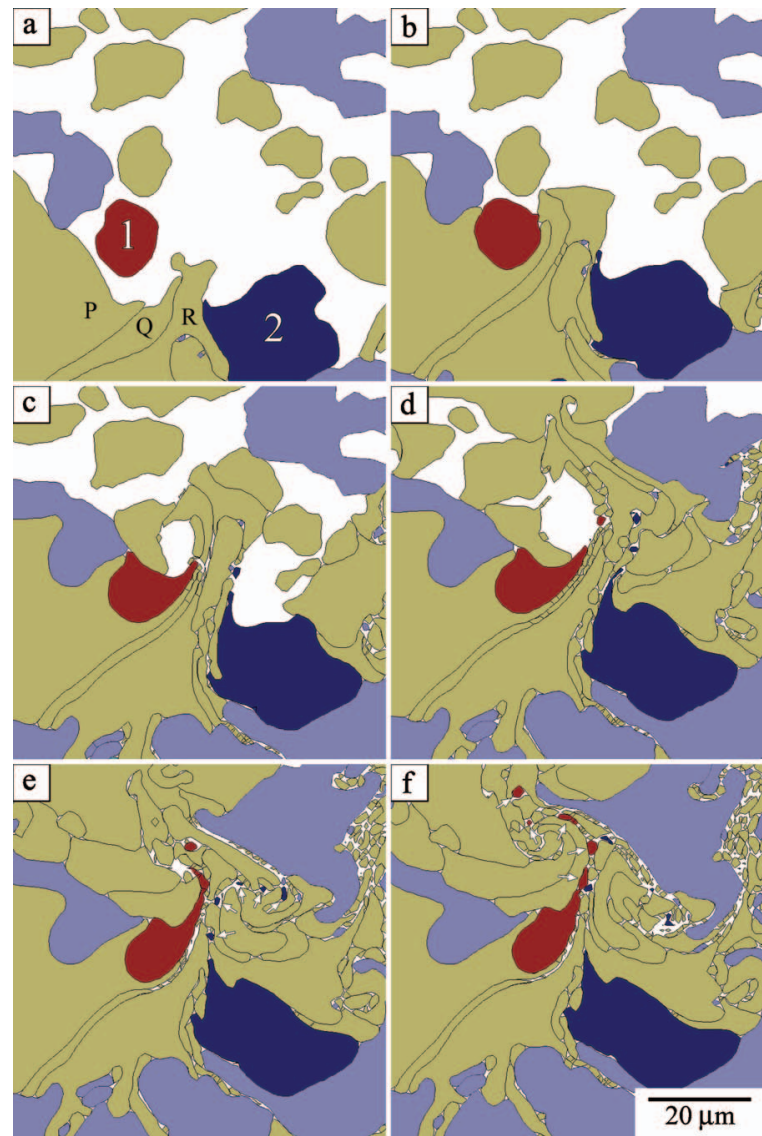

FIG. 12. (Color) Series of snapshots showing particulation of Ni particles 1 and 2 caused by cooperative focused flow of aluminum particles P, Q, and $\mathrm{R}$. Note the dispersion of the $\mathrm{Ni}$ particulates over distances of a particle diameter from their parent particles. Time between frames is $8 \mathrm{~ns}$.

sumes a continuous particle velocity across material interfaces, prohibiting particle sliding, but allowing the pulling, necking, and dispersion of nickel particulates into the aluminum flow. These particulates [indicated by the arrows in Figs. 12(e) and 12(f)] are severely strained, quickly attain high temperatures, and can become separated from their parent particle dispersed over distances of several particle diameters.

The simultaneous development of multiple focused flows throughout a highly irregular high-pressure front can in some cases lead to the formation of "vortices" within the flow channels, as shown in the sequence of images in Fig. 13. Though it is slightly more challenging to generalize the events leading to vortex formation, their effects on deformation and mass flow are singular. Vortices are locations of tremendous strain, large opposing particle velocities, and local temperatures. Focused flow and vortex formation are attributed and often observed to produce the wave structures at interfaces between clad plates during explosive welding. ${ }^{46,47}$ Even after the passage of the high-pressure front, the inertia of the vortices keeps them in motion far longer than the surrounding material (up to $\sim 100$ and $\sim 60$ ns behind the high-pressure front in the case of the $45 \%$ and $80 \%$ TMD simulations, respectively, with $U_{p}=1 \mathrm{~km} / \mathrm{s}$ ). The sequence of images in Fig. 13 show an example illustrating the closure of a vortex located at the head of the high-pressure front in 


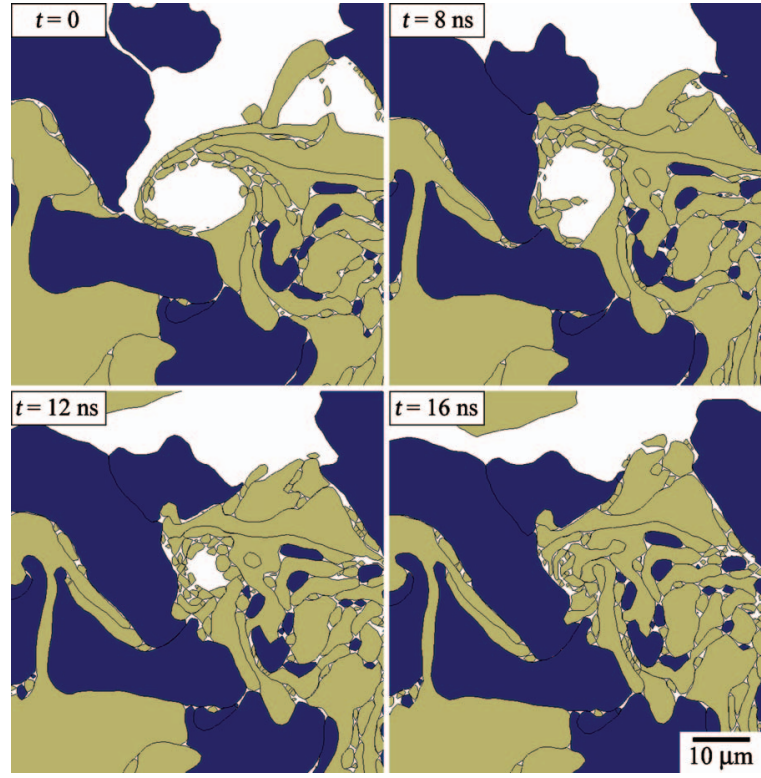

FIG. 13. (Color) Sequence of images showing a deformation "vortex" (and its closure) caused by flow instability at the head of the shock front in the $45 \% \mathrm{TMD} \mathrm{Ni}+\mathrm{Al}$ simulation.

the $45 \%$ TMD simulation $\left(U_{p}=1 \mathrm{~km} / \mathrm{s}\right)$. Though the aluminum exhibits tremendous particulation within the vortex after $16 \mathrm{~ns}$, the precise number and size of particulates observed are attributed to the mesh size. While vortices are transient phenomena, their signatures have been also observed in microstructures of recovered shock-compressed materials. Shown in Fig. 14 are patterns attributed to vortex flow in the $\mathrm{SEM}$ micrograph of a recovered $60 \%$ dense $\mathrm{Ni}+\mathrm{Al}$ specimen impacted at $980 \mathrm{~m} / \mathrm{s}^{39}$ Note also the evidence of the grayish-contrast second phases, which suggests that the strains, temperatures, and associated local mass mixing within vortices may be sufficient to trigger local chemical reactions.

The frequency of the processes described above largely depends on void content. At higher initial densities, singular focused flows dominate, and the occurrence of cooperative focused flows, and correspondingly particulation and vortex formation, decreases.

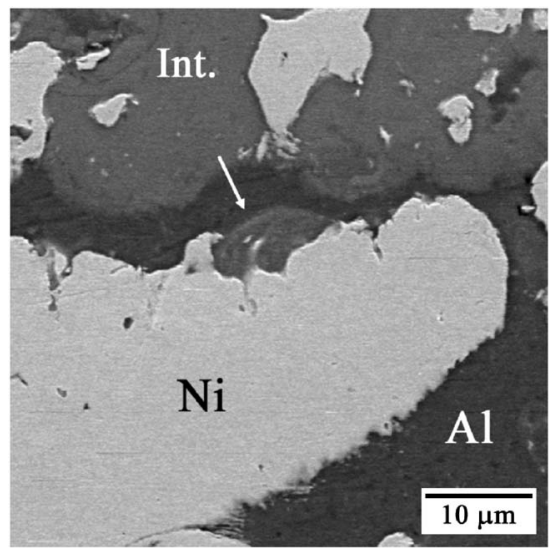

FIG. 14. SEM image showing the signature of a vortex in a $60 \%$ TMD $\mathrm{Ni}+\mathrm{Al}$ mixture recovered following shock compression of sample impacted at $980 \mathrm{~m} / \mathrm{s}$. Note the formation of a grayish-contrast intermetallic phase at the vortex site and in the surrounding areas.

\section{DISCUSSION}

Simulations of shock wave propagation through highly porous solids using real microstructures of $\mathrm{Ni}+\mathrm{Al}$ powder mixture compacts have provided a clear picture regarding the role of void content on the shock compression of mixtures of powders of dissimilar density and strength. The development of stable pressures is intimately linked to the amount of void volume available for particle motion and deformation. Limited porosity at high initial densities results in quickly collapsed voids, rapid increase in local pressure at confined particles, and sustained high-pressure state. On the other hand, mixtures of powders packed at low densities experience enhanced particle deformation through several modes, in addition to the associated development of high local temperatures, longer void closure times, and dispersion of the apparent shock front caused by release pockets.

The high-pressure disturbances observed in this work do not possess the characteristics of classical shocks, as typically seen in homogeneous media. The existence of a precursor disturbance at intermediate to high densities (Fig. 6) is reminiscent of underdriven shocks, at best. Furthermore, the instabilities noted behind the high-pressure front (Figs. 7-9) indicate a significant departure from mechanical and thermal equilibrium, suggestive of the more familiar notion of catastrophic shocks. ${ }^{25}$ Such behavior, albeit to a lesser degree, has been discussed even in the case of solid materials. ${ }^{48,49}$ Heterogeneities in structure arising from grain boundaries in polycrystalline materials result in the formation of varied states of deformation. It is no surprise then that the enhanced heterogeneity of granular systems produces extreme localized deformation, resulting in very complex, multidimensional disturbances. These observations imply that under the conditions investigated, a classical shock does not exist in the highly heterogeneous $\mathrm{Ni}+\mathrm{Al}$ powder mixture system. The conditions for a uniform, steady-state shock might be approached at significantly higher loading conditions, where the mechanical instabilities behind the high-pressure front equilibrate more rapidly.

The validity of the simulations has been established for the 60\% TMD simulation data which shows a good match with available experimental data. It should be noted that the most important detail omitted in the present two-dimensional simulations is the influence of out-of-plane particle contacts. Such contacts may assist in extending either the lowamplitude stress network or high-pressure front, and might possibly bear a greater role as the initial mixture density decreases. Serial sections imported for three-dimensional simulations are in progress to evaluate the influence of such out-of-plane contacts and further establish the heterogeneous nature of shock propagation in powder mixtures, as well as their influence on physical and chemical changes.

\section{CONCLUSIONS}

The mechanics of shock compression and particle-level deformation have been investigated through Eulerian finite element simulations using CTH. Experimentally acquired micrographs of equivolumetric $\mathrm{Ni}+\mathrm{Al}$ powder mixtures at varying densities have been used to preserve the highly ir- 
regular configurations often ignored by idealized, artificially generated techniques. The influence of density on the mixture equation of state was determined and validated with previously obtained experimental data at $60 \%$ TMD. Tracking of the bulk shock front at multiple locations revealed that the shock velocity was constant over a $1.5 \mathrm{~mm}$ distance, which justifies the coupling of experimentally measured input stress and propagated shock velocity.

Greater insight into the inhomogeneous nature of shock compression of deformable granular media was gained though examination of particle-scale dynamics and accumulative material response at multiple scales. Pressure surfaces constructed for the various mixtures revealed the unstable character of low-amplitude stress and high-pressure fronts at low densities, and indicated pressure equilibration times of up to $100 \mathrm{~ns}$ after arrival. These effects were used to describe dispersion on the particle level. The initial density exhibited direct influence over the local pressures and temperatures developed due to the lack and presence of flow channels, respectively. The dominant heterogeneous deformation mechanisms leading to focused flow, particulation, and vortexing were also cataloged and attributed to local fluctuations in pressure and temperature, which collectively are responsible for highly irregular shock fronts and unstable highpressure states in granular materials. While it is challenging to probe these effects in real time, discrete particle-level simulations performed on heterogeneous systems using real (imported) microstructures provide valuable details of heterogeneous deformation processes leading to the observed macroscopic phenomena.

\section{ACKNOWLEDGMENTS}

The authors wish to acknowledge funding provided by AFOSR/MURI Grant No. F49620-02-1-0382 and through a National Defense Science Engineering Graduate Fellowship awarded to one of the authors (D.E.). The multimaterial Eulerian finite element code CTH used in the present work was developed at Sandia National Laboratories (SNL). The authors acknowledge SNL for permission to use this code and thank Dr. David Crawford for providing the training for its usage.

${ }^{1}$ S. M. Sweeney and C. L. Martin, Acta Mater. 51, 3635 (2003).

${ }^{2}$ A. Perez-Fouguet, A. Rodriguez-Ferran, and A. Huerta, Comput. Mech. 30, 220 (2003).

${ }^{3}$ D. T. Gethin, R. W. Lewis, and R. S. Ransing, Modell. Simul. Mater. Sci. Eng. 11, 101 (2003).

${ }^{4}$ A. T. Procopio and A. Zavaliangos, J. Mech. Phys. Solids 53, 1523 (2005).

${ }^{5}$ F. X. Sanchez-Castillo, J. Anwar, and D. M. Heyes, Chem. Mater. 15, 3417 (2003).

${ }^{6}$ Z. Q. Jin et al., J. Mater. Res. 20, 599 (2005).

${ }^{7}$ X. Xiao and N. N. Thadhani, TMS Annual Meeting and Exhibition of Minerals, Metals and Materials Society, Nashville, TN, 2000, p. 23, ISSN No.1359-6462.

${ }^{8}$ R. Prummer and P. Weimar, Interceram 51, 394 (2002).

${ }^{9}$ A. N. Dremin and O. N. Breusov, Russ. Chem. Rev. 37, 392 (1968).

${ }^{10}$ N. N. Thadhani, J. Appl. Phys. 76, 2129 (1994).

${ }^{11}$ R. A. Graham, Proceedings of the Third International Symposium on High Dynamic Pressures, edited by R. Charet (La Grande-Motte, France, 1989), p. 175.

${ }^{12}$ J. E. Flinn, R. L. W. R. A. Berry, R. N. Wright, Y. M. Gupta, and M. Williams, J. Appl. Phys. 64, 1446 (1988).
${ }^{13}$ M. Kamegai, O. R. Walton, and A. G. Taylor, in Shock Compression of Condensed Matter, edited by S. C. Schmidt, J. N. Johnson, and L. W. Davison (North-Holland, Albuquerque, 1989), p. 567.

${ }^{14}$ D. J. Benson, W. Tong, and G. Ravichandran, Modell. Simul. Mater. Sci. Eng. 3, 771 (1995)

${ }^{15}$ D. J. Benson, Wave Motion 21, 85 (1995).

${ }^{16}$ T. G. Nieh, P. Luo, W. Nellis, D. Lesuer, and D. Benson, Acta Mater. 44, 3781 (1996).

${ }^{17}$ Z. P. Tang, Y. Horie, and S. G. Psakhie, in High-Pressure Shock Compression of Solids IV: Response of Highly Porous Solids to Shock Loading, edited by L. Davison, Y. Horie, and M. Shahinpoor (Springer, New York, 1997), p. 143.

${ }^{18}$ D. J. Benson and P. Conley, Modell. Simul. Mater. Sci. Eng. 7, 333 (1999).

${ }^{19}$ Y. Horie and K. Yano, J. Mater. Process. Technol. 85, 109 (1999).

${ }^{20}$ M. R. Baer and W. M. Trott, in Shock Compression of Condensed Matter, edited by M. D. Furnish, Y. M. Gupta, and J. W. Forbes (American Institute of Physics, Portland, OR, 2003), p. 517.

${ }^{21}$ R. A. Austin, D. L. McDowell, and D. J. Benson, Modell. Simul. Mater. Sci. Eng. 14, 537 (2006)

${ }^{22}$ E. M. Bringa et al., Nat. Mater. 5, 805 (2006).

${ }^{23}$ S. G. Bardenhagen and J. U. Brackbill, J. Appl. Phys. 83, 5732 (1998).

${ }^{24}$ S. J. Antony, M. R. Kuhn, D. C. Barton, and R. Bland, J. Phys. D 38, 3944 (2005).

${ }^{25}$ R. A. Graham, J. Phys. Chem. 83, 3048 (1979).

${ }^{26}$ R. L. Williamson, J. Appl. Phys. 68, 1287 (1990)

${ }^{27}$ D. R. Kumar, R. K. Kumar, and P. K. Philip, J. Appl. Phys. 85, 767 (1999).

${ }^{28}$ M. R. Baer, in Shock Compression of Condensed Matter, edited by M. D. Furnish, L. C. Chhabildas, and R. S. Hixson (American Institute of Physics, Snowbird, UT, 1999), p. 27.

${ }^{29}$ A. Al-Omari and E. Masad, Int. J. Numer. Analyt. Meth. Geomech. 28, 1327 (2004).

${ }^{30}$ A. D. Jadhav, N. P. Padture, E. H. Jordan, M. Gell, P. Miranzo, and E. R. Fuller, Jr., Acta Mater. 54, 3343 (2006).

${ }^{31}$ T. Kanit, F. N'Guyen, S. Forest, D. Jeulin, M. Reed, and S. Singleton, Comput. Methods Appl. Mech. Eng. 195, 3960 (2006).

${ }^{32}$ J. Wulf, T. Steinkopff, and H. F. Fischmeister, Acta Mater. 44, 1765 (1996).

${ }^{33}$ J. Zhai, V. Tomar, and M. Zhou, J. Eng. Mater. Technol. 126, 179 (2004).

${ }^{34}$ S. D. Bona, L. Lutzemberger, and O. Salvetti, Phys. Med. Biol. 48, 4001 (2003).

${ }^{35}$ A. Gefen, M. Medigo-Ravid, Y. Itzchak, and M. Arcan, Proceedings of The First Joint BMES/EMBS Conference, Atlanta, GA, 1999, p. 559, ISSN No. 0589-1019.

${ }^{36} \mathrm{CTH}$, v. 7.1, Sandia National Laboratories.

${ }^{37}$ Raster to Vector Conversion Toolkit, v. 2.97.3, Algolab Inc.

${ }^{38}$ D. J. Benson, Modell. Simul. Mater. Sci. Eng. 2, 535 (1994).

${ }^{39}$ D. E. Eakins and N. N. Thadhani, in Shock Compression of Condensed Matter, edited by M. D. Furnish, M. Elert, T. P. Russell, and C. T. White (American Institute of Physics, Baltimore, MD, 2005), p. 1153.

${ }^{40}$ D. J. Steinberg, S. G. Cochran, and M. W. Guinan, J. Appl. Phys. 51, 1498 (1980).

${ }^{41}$ G. R. Johnson and W. H. Cook, Proceedings of the Seventh International Symposium on Ballistics (ADPA, The Netherlands, 1983), p. 541.

${ }^{42}$ J. P. Borg, D. J. Chapman, K. Tsembelis, W. G. Proud, and J. R. Cogar, J. Appl. Phys. 98, 73509 (2005).

${ }^{43}$ D. E. Eakins and N. N. Thadhani, in 2005 Materials Research Society Fall Meeting, edited by N. N. Thadhani, R. W. Armstrong, A. E. Gash, and W. H. Wilson (Materials Research Society, Warrendale, PA, 2005), p. 191.

${ }^{44}$ M. U. Anderson, R. A. Graham, and G. T. Holman, International Conference of the International Association for the Advancement of High Pressure Science and Technology (Colorado Springs, Colorado, 1993), p. 1111, ISSN No. 0094-243X

${ }^{45}$ X. Xu and N. N. Thadhani, J. Appl. Phys. 96, 2000 (2004).

${ }^{46}$ S. L. Wang, M. A. Meyers, and A. Szecket, J. Mater. Sci. 23, 1786 (1988).

${ }^{47}$ E. A. Kozlov, A. V. Dobromyslov, B. V. Litvinov, N. I. Taluts, G. V. Kovalenko, G. G. Bondarchuk, and G. V. Dolgikh, Phys. Met. Metallogr. 95, 200 (2003).

${ }^{48}$ J. R. Asay and L. C. Chhabildas, in High-Pressure Shock Compression of Solids VI: Old Paradigms and New Challenges, edited by Y. Horie, L. Davison, and N. N. Thadhani (Springer, New York, 2002), p. 57.

${ }^{49}$ M. A. Meyers and M. S. Carvalho, Mater. Sci. Eng. 24, 131 (1976). 